\title{
The Sense of Presence through the Humanization Created by Virtual Environments ${ }^{\dagger}$
}

\author{
João Donga 1,2,*, António Marques ${ }^{2}$, Javier Pereira ${ }^{3}$ and Paulo Veloso Gomes ${ }^{2}$ \\ 1 ESMAD, Escola Superior de Media Artes e Design do Politécnico do Porto, \\ 4480-876 Vila do Conde, Portugal \\ 2 LabRP, Laboratório de Reabilitação Psicossocial, Escola Superior de Saúde do Politécnico do Porto, \\ 4200-072 Porto, Portugal \\ 3 CITIC-Research Center of Information and Communication Technologies, University of A Coruña, \\ 15071 A Coruña, Spain \\ * Correspondence: jpd@esmad.ipp.pt \\ + Presented at the 2nd XoveTIC Conference, A Coruña, Spain, 5-6 September 2019. \\ Published: 23 July 2019
}

\begin{abstract}
This work focus on the study of solutions that using video 360 and virtual reality that allow children's and older people that are away of their family environments for various reasons to be able to feel they are participating at family or school events. The solutions proposed should deliver a strong sense of presence to the users and the interface must be friendly. The validation will be made by user observation and inquiries.
\end{abstract}

Keywords: video 360; virtual reality; telepresence; embodiment; presence; augmented human; head-mounted display; immersion; user experience

\section{Introduction}

Nowadays, with increasing life expectancy, there are more and more elderly people living alone in their homes or in institutions. Loneliness is one of the main problems affecting this community and there are various means of communication available to communicate with family and friends. This study seeks to create communication mechanisms that allow a high sense of presence.

\section{Discussion}

The solutions currently used to allow the communication between target users (elderly people) and the outside are based on the use of the mobile phone or the use of videoconferencing mechanisms using laptops or smartphone apps. We intend to study the extent to which these solutions offer a satisfactory sense of presence and create alternative solutions using video 360 to increase this sense of presence to more satisfactory levels (Figure 1).

Presence is the feeling or experience of "being" in the VR environment [1]. Witmer and Singer [2] define presence as "the subjective experience of being in one place or environment, even when one is physically situated in another". Often confused, the terms immersion and presence should not be considered has equivalent terms. Mel Slater defines the terms this way [3]:

- Immersion refers to the objective level of sensory fidelity a VR system provides.

- Presence refers to a user's subjective psychological response to a VR system. 


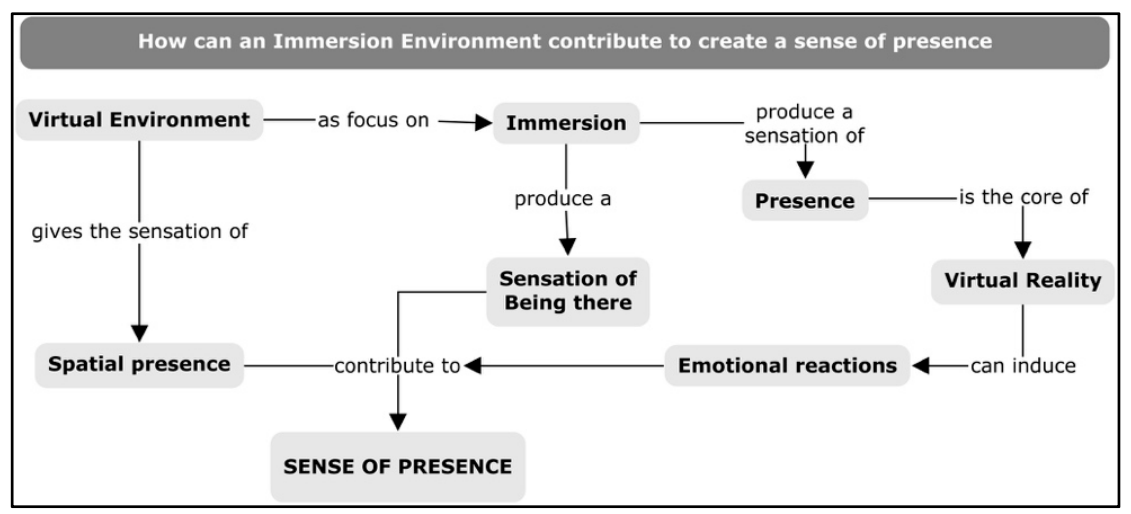

Figure 1. A graphic illustration of the contribution of immersive environments to create a sense of presence.

To create a VR solution with a strong sense of presence we have identified several aspects and we divide them in three main categories: emotion, environment and communication (Figure 2).

- Environmental presence is perception that a virtual environment exists and that the user is present within it [4]. A high degree of environmental presence will increase immersion of virtual experience.

- Communication and interaction with others in virtual reality is very important component for social presence [5].

- Presence has been found to be mediated by human, context, and medium characteristics. Amongst the human factors, a possible relationship between presence and emotions has been noted [6].

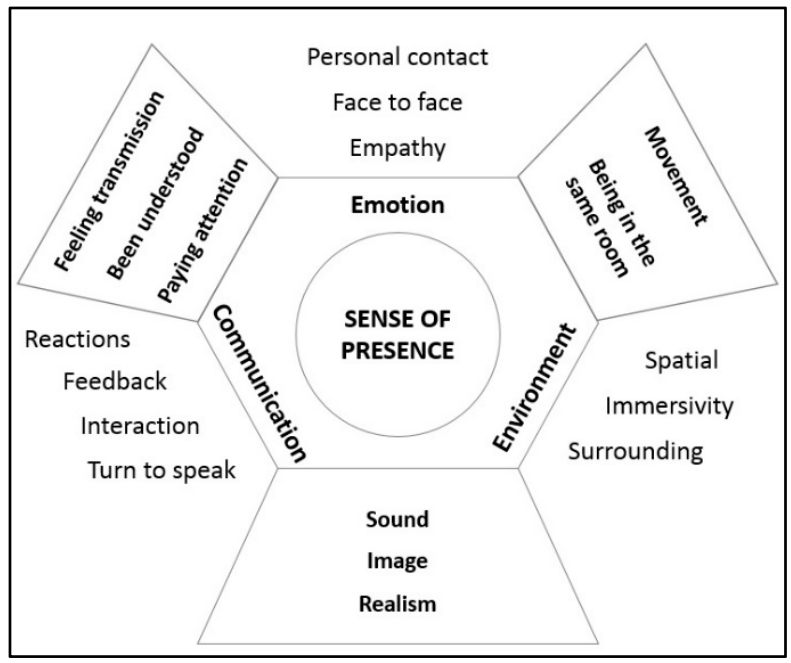

Figure 2. A graphic illustration of the relationship between emotion, communication and environment and their relation with sense of presence.

Cinematic Virtual Reality is argued to hold the potential to provide a more immersive viewing experience, in which the user feels a sense of presence, or a feeling of "being there", similar to traditional computer- generated imagery based VR [7].

Previous research also shows mixed effects about sense of presence in $360^{\circ}$ video. MacQuarrie and Steed found that watching $360^{\circ}$ videos in a head-mounted display (HMD) created a stronger sense of presence than a surround video display or a regular TV and less concern about missing out on content [8]. 
In the first phase, the work uses the Research-Action Methodology to design a model for the construction of the technological artefact, prepare the construction of the prototype and the tests to be implemented, identify the relevant indicators and prepare its application.

The second phase applies the developed model to specific cases, using specific Focus Groups. The third phase focuses on evaluation of the impact of intervention and results discussion. This evaluation will be made using questionnaires to measure the sense of presence, usability and user interface.

Author Contributions: Conceptualization, J.D.; methodology, J.D. and A.M.; validation, J.D. and P.V.G.; investigation, J.D.; writing - original draft preparation, J.D.; writing - review and editing, J.D.; visualization, J.D. and P.V.G; supervision, A.M. and J.P.; project administration, J.D.

Funding: This research received no external funding.

Conflicts of Interest: The authors declare no conflict of interest. The funders had no role in the design of the study; in the collection, analyses, or interpretation of data; in the writing of the manuscript, or in the decision to publish the results.

\section{References}

1. Wallach, H.S.; Safir, M.P.; Samana, R.; Almog, I.; Horef, R. How Can Presence in Psychotherapy Employing VR Be Increased? Chapter for Inclusion in: Systems in Health Care Using Agents and Virtual Reality. In Advanced Computational Intelligence Paradigms in Healthcare 6. Virtual Reality in Psychotherapy, Rehabilitation, and Assessment; Brahnam, S., Jain, L.C., Eds.; Springer: Berlin/Heidelberg, Germany, 2011; Chapter 7, pp. 129-147, doi:10.1007/978-3-642-17824-5_7.

2. Witmer, B.G.; Singer, M.J. Measuring presence in virtual environments: A presence questionnaire. Presence $1998,7,225-240$.

3. Slater, M. A note on presence terminology. Presence Connect 2003, 3, 1-5.

4. A Situated Cognition Perspective on Presence. Available online: https://www.researchgate.net/publication/ 28764862_A_situated_cognition_perspective_on_presence (accessed on 14 July 2019).

5. Short, J.A.; Williams, E.; Christie, B. The Social Psychology of Telecommunications; John Wiley \& Sons: New York, NY, USA, 1976.

6. Banos, R.M.; Botella, C.; Liano, V.; Guerrero, B.; Rey, B.; Alcañiz, M. Sense of presence in emotional virtual environments. In Proceedings of the Presence 2004: The 7th Annual Workshop on Presence, Valencia, Spain, 13-15 October 2004; pp. 156-159.

7. Aitamurto, T.; Zhou, S.; Sakshuwong, S.; Saldivar, J.; Sadeghi, Y.; Tran, A. Sense of Presence, Attitude Change, Perspective-Taking and Usability in First-Person Split-Sphere $360^{\circ}$ Video. In Proceedings of the 2018 CHI Conference on Human Factors in Computing Systems, Montreal, QC, Canada, 21-26 April 2018; pp. 1-12, doi:10.1145/3173574.3174119

8. MacQuarrie, A.; Steed, A. Cinematic virtual reality: Evaluating the effect of display type on the viewing experience for panoramic video. In Proceedings of the 2017 IEEE Virtual Reality (VR), Los Angeles, CA, USA, 18-22 March 2017. pp. 45-54.

(c) 2019 by the authors. Licensee MDPI, Basel, Switzerland. This article is an open access article distributed under the terms and conditions of the Creative Commons Attribution (CC BY) license (http://creativecommons.org/licenses/by/4.0/). 